\title{
Recurrent pancreatitis induced by hyperparathyroidism in pregnancy
}

\author{
Robert Krysiak $\cdot$ Mariusz Wilk $\cdot$ Bogusław Okopien
}

Received: 5 May 2010 / Accepted: 25 August 2010 / Published online: 17 September 2010

(C) The Author(s) 2010. This article is published with open access at Springerlink.com

\begin{abstract}
Introduction As primary hyperparathyroidism affects mainly middle-aged and elderly women, it is an infrequent finding during gestation and breastfeeding. To date, less than 200 pregnant patients with primary hyperparathyroidism diagnosed during pregnancy have been described. Like in other disorders of the parathyroid gland, the recognition of primary hyperparathyroidism during pregnancy and lactation may be difficult, as clinical symptoms are not specific, while laboratory findings may be masked by some typical pregnancy-induced changes in calcium and phosphate homeostasis. If remains untreated, the disease may result in serious clinical implications for the mother and fetus. Most authors consider surgery within the second trimester of pregnancy as the treatment of choice in this group of patients.

Case report In our paper, we discuss the case of a 35-year-old female with a history of recurrent acute pancreatitis and recurrent abortions. As the patient declined surgery, conservative management with calcitonin was started and continued throughout the rest of pregnancy, and led to giving birth to the infant whose only health problem was transient hypocalcemia.

Conclusion The described case shows that conservative management, if started respectively early and conducted on the basis of a patient's condition, may effectively reduce increased perinatal and maternal morbidity and mortality in pregnant women declining surgery.
\end{abstract}

R. Krysiak $(\bowtie) \cdot$ B. Okopien

Department of Internal Medicine and Clinical Pharmacology,

Medical University of Silesia, Medyków 18,

40-752 Katowice, Poland

e-mail: r.krysiak@interia.pl

M. Wilk

Department of Histopathology,

Medical University of Silesia, Katowice, Poland
Keywords Primary hyperparathyroidism .

Calcium homeostasis · Pregnancy and postpartum period .

Clinical course $\cdot$ Diagnosis and management

\section{Introduction}

In the general population, primary hyperparathyroidism is considered a common disorder. Its greatest frequency is observed in postmenopausal women, in whom it reaches a prevalence of $2-3 \%$ [1]. The most frequent cause of primary hyperparathyroidism in the general population is a single parathyroid adenoma ( $85 \%)$, followed by parathyroid hyperplasia (15-20\%) and, albeit very rarely (less than $1 \%$ ), by carcinoma $[2,3]$. Interestingly, less than 200 of patients with primary hyperparathyroidism have been described in gestation and during the postpartum period [4-6]. This relative paucity of data may be explained by at least three different causes. Firstly, the average age of the initial manifestation of this disorder is higher than that of women of childbearing age [1-3]. Second, about $80 \%$ of non-pregnant individuals with hyperparathyroidism are characterised by an asymptomatic course of this disease [5]. And finally, some symptoms of primary hyperparathyroidism may be misinterpreted as a simple consequence of pregnancy or other gestation-related disorders, while physiological changes during gestation may mask some abnormalities typical to primary hyperparathyroidism [4, 7]. What is worth mentioning is that compared to the remaining subjects, pregnant ones with primary hyperparathyroidism often experience a clinically overt course of this disease [5, 6]. Making the correct diagnosis of primary hyperparathyroidism in pregnancy is regarded crucial, because if this disorder remains unrecognized and left untreated, it may pose a significant risk to the mother and 
fetus, which is associated with increased perinatal and maternal morbidity and mortality $[6,8]$.

This article shows the case of a young woman with three spontaneous abortions, in whom primary hyperparathyroidism was diagnosed for the first time during fourth gestation. In the paper, we present diagnostic and treatment dilemmas associated with finding and treatment of this disorder during gestation. We describe in detail treatment strategies undertaken in this patient and their impact on the outcome of pregnancy and lactation.

\section{Clinical picture}

A 35-year-old woman was admitted to the gastroenterological ward in the eighth week of her fourth pregnancy because of a strong abdominal pain, nausea, vomiting and anorexia. All her previous pregnancies ended in spontaneous miscarriages in the, respectively, tenth, eighth and sixth week of gestation. During the last one, taking place a year before the present admission, the abortion was preceded by laboratoryconfirmed acute pancreatitis. In the second gestation, she experienced similar symptoms of a strong periumbilical pain and vomiting. These symptoms, which occurred a week before pregnancy termination and treated without broadening diagnosis, retrospectively suggested the presence of pancreatitis. On admittance to the gastroenterological ward, the patient presented symptoms of dehydratation. She was asthenic, had dry skin and mouth. The thyroid gland was not palpable and there was no galactorrhea. Abdomen was distended and the bowel sounds were absent. The liver was slightly enlarged $(2 \mathrm{~cm}$ below the costal margin). Uterine size corresponded to the period of gestation. Blood pressure was reduced $(90 / 65 \mathrm{mmHg})$, heart rate was 104 beats $/ \mathrm{min}$, while temperature was elevated (and $38.2^{\circ} \mathrm{C}$ ). She had no history of gestational diabetes mellitus, alcohol use, oral contraception pills, gallstones or drug intake. Initial laboratory investigations revealed increased amylasaemia $(825 \mathrm{U} / \mathrm{L}$; normal values $<150 \mathrm{U} / \mathrm{L})$ and lipasaemia $(980 \mathrm{U} / \mathrm{L}$; normal values $<150 \mathrm{U} / \mathrm{L})$, increased urine amylase content $(420 \mathrm{U} / \mathrm{L}$; normal range $8-12 \mathrm{U} / \mathrm{L})$, hyperglycemia $(7.4 \mathrm{mmol} / \mathrm{L})$, increased leucocyte count $\left(19,800 / \mathrm{mm}^{-3}\right)$, increased CRP levels $(122 \mathrm{mg} / \mathrm{L}$; normal values $<5 \mathrm{mg} / \mathrm{L})$ and a hematocrit of $47.8 \%$. Lipid profile and liver and renal function tests (plasma activity/levels of alanine aminotransferase, aspartate aminotransferase, $\gamma$-glutamyl transpeptidase, total bilirubin, creatinine and urea nitrogen) were within normal limits. On ultrasound the patient had oedematous pancreatitis with irregular pancreatic outline, and a small amount of peripancreatic fluid as well as bilateral massive nephrolithiasis without evidence of cholelithiasis. Computed tomography was not performed (contraindicated in pregnancy), and the patient did not undergo magnetic resonance imaging examination due to a suggestive clinical picture and the lack of agreement (a fear of thermal damage of fetal tissues). The patient was diagnosed with acute pancreatitis without systemic complications (Ranson's score 2, APACHE II score 4, BISAP 1) and treated conservatively with fasting, narcotic analgesia (pethidine $50 \mathrm{mg}$ subcutaneously every $6 \mathrm{~h}$ for the first $24 \mathrm{~h}$ ) and intravenous fluids. Because of concomitant asymptomatic bacteriuria she also received cefuroxime $(750 \mathrm{mg}$ intramuscularly every $8 \mathrm{~h}$ for 5 days). Although during this treatment her physical condition has markedly improved, initially highnormal calcaemia (plasma calcium levels corrected for albumin $2.6 \mathrm{mmol} / \mathrm{L}$; normal values $2.2-2.6 \mathrm{mmol} / \mathrm{L}$, ionized calcium $1.3 \mathrm{mmol} / \mathrm{L}$; normal values $0.9-1.3 \mathrm{mmol} / \mathrm{L}$ ) started to grow reaching on day 4 the following values: total calcium $3.2 \mathrm{mmol} / \mathrm{L}$, ionized calcium $1.7 \mathrm{mmol} / \mathrm{L}$. This was accompanied by hypophosphatemia $0.7 \mathrm{nmol} / \mathrm{L}$ (normal values $0.9-1.5 \mathrm{mmol} / \mathrm{L}$ ) and therefore the patient was transferred to our unit. On admittance to our ward she had elevated plasma intact PTH assessed using immunoradiometric assay (130 ng/L; normal values $15-75 \mathrm{ng} / \mathrm{L}$ ), while 25 -hydroxyvitamin D plasma levels $(25 \mathrm{nmol} / \mathrm{L})$ were within the normal limits $(15-80 \mathrm{nmol} / \mathrm{L})$. The levels of serum amylase and lipase were within the reference values. Because of the pregnancy we did not decide on the measurement of alkaline phosphatase, is increased during normal gestation. No abnormalities in plasma levels of thyrotropin, free thyroxine and free triiodothyronine were observed. As these results suggested the presence of primary hyperpararthyroidism, we introduced intavenous infusions of saline and the patient was managed with salmon calcitonin (at the dose of $4 \mathrm{mg} / \mathrm{kg}$ administered intramuscularly every $8 \mathrm{~h} ; 12$ doses), which is more potent and longer acting than the human hormone. We also suggested that the patient should undergo surgical intervention, preferably in the second trimester of pregnancy, as a radical treatment. After informing the patient about benefits and risks associated with the procedure, she declined this form of therapy during pregnancy, agreeing on its performance after delivery. Calcitonin treatment resulted in a clinical improvement and quick resolving of hypercalcemia, while ultrasonography revealed normal fetal development without growth retardation, the patient was discharged from hospital on twelfth week of pregnancy and recommended to monitor plasma calcium and phosphate levels on a weekly basis. She was advised to take about $4 \mathrm{~L}$ of fluids daily to increase calcium excretion. In case of symptoms of hypercalcemia (constipations, proximal muscle weakness, fatigue, anorexia, abdominal pains, depression or any cognitive disfunction) or increased plasma calcium levels exceeding $2.8 \mathrm{mmol} / \mathrm{L}$ she was suggested to contact our department to modify hypercalcemia treatment. Between weeks 12 and 32 she periodically experienced constipations or muscle weakness, which were accompanied by an increase in plasma calcium levels and resolved after intramuscular injections of 
calcitonin. During these 20 weeks she received calcitonin 14 times. Because since week 32, the need of calcitonin administration became more evident (between weeks 32 and 34 she had to receive calcitonin twice a week with only partial clinical response), we decided to recommend our patient $3 \mathrm{~g}$ of oral phosphates, which resulted in a lowering calcium levels and increasing phosphate levels to the values observed in healthy subjects. The gestation ended in the 38th week with spontaneous vaginal delivery. A live-born infant $(3,250 \mathrm{~g}$, $52 \mathrm{~cm}$ ) had Apgar score of 9 without any malformations observed on physical examination. The infant was found, however, to have hypercalcemia $(2.9 \mathrm{mmol} / \mathrm{L})$ immediately after birth. Initially increased calcium levels started to decrease in the following days reaching on neonatal day 6 the value of $1.9 \mathrm{mmol} / \mathrm{L}$. Because of the risk of symptomatic hypoparathyroidism we started intravenous calcium infusion (400 mg/kg/day of calcium gluconate), which led to a normalisation of plasma calcium during $6 \mathrm{~h}$. The infusion was continued for the 4 following days, since neonate day 8 with gradually reduced rate and on day 10 it was finally discontinued. The child had no other health problems related to primary hyperpaprathyroidism and therefore was discharged from hospital on day 14. Subsequent follow ups revealed its normal development. Cervical computed tomography, performed 2 months after delivery, revealed the presence of a nodule located posteriorly to the upper part of the left thyroid lobe, in which $99 \mathrm{mTc}$-sestamibi was clearly accumulated. Three months after delivery, the patient underwent surgery for the excision of this tumor. Since delivery to the time of operation the subject did not need to be given calcitonin or phosphates and her calcium levels were slightly elevated (total calcium $2.9 \mathrm{mmol} / \mathrm{L}$, ionized calcium $1.5 \mathrm{mmol} / \mathrm{L}$. Histological examination of the removed tissue confirmed the presence of a benign adenoma of the parathyroid gland. Because of nephrolithiasis, 6 month after delivery she underwent lithotripsy. Two years later, the patient became pregnant for the fifth time. The course of this pregnancy was uneventful and ended in a spontaneous delivery in 40 week, with no abnormalities in the infant.

\section{Discussion}

Pregnancy and lactation are characterized by important alterations in calcium homeostasis, being a consequence of pregnancy-induced changes in the synthesis, metabolism and excretion of calcium and calcitropic hormones [4, 9]. For these reasons clinical manifestations of parathyroid disorders in pregnancy are often different from those observed in non-pregnant women. As the symptoms experienced by patients with parathyroid disorders are not specific, their diagnosis during gestation and breastfeeding may be sometimes very difficult $[4,9]$. Unfortunately this was the case in our patient, whose three previous pregnancies, because of the presence of unrecognized parathyroid pathology, ended in intrauterine fetal demise, which in two pregnancies was preceded by documented intrauterine growth retardation. These results clearly indicate that primary hyperparathyroidism, if remains undiagnosed and consequently untreated, is associated with a markedly increased risk of pre- and perinatal mortality.

The data obtained from analysis of so far described cases indicate that in opposition to the general population, 4 of every 5 hyperparathyroid pregnant patients experience clinical manifestations of this disorder. The most frequent of them, observed also in our patient, was the presence of nephrolithiasis [5]. The initiation and growth of kidney calculi may be attributed to overlapping of both increased calcium load, secondary to enhanced PTH synthesis and release, and a pregnancy-induced increase in urine calcium excretion (a typical symptom of physiological pregnancy). The large size of concrements in our patient may be explained by her undergoing several pregnancies during a relative short period of time.

However, the most characteristic health problem experienced by the patient was recurrent acute pancreatitis. Interestingly, the frequency of this complication in pregnancyrelated primary hyperparathyroidism is higher (7-13\%) than in hyperparathyroidism taking place in non-pregnant individuals $(1-2 \%)[5,10,11]$. The fact that pancreatitis was present in three of four pregnancies complicated by hyperparathyroidism, while never occurred before and between pregnancies, supports these statistical data that gestation makes hyperparathyroid patients particularly prone to the development of this complication. It is assumed that pancreatitis occur more frequently in primiparas than in women who underwent multiple pregnancies and occurs mainly in the first and third trimester of gestation $[6,12]$. Our observations only partially support these data. Although all cases of pancreatitis in our subject occurred in the first trimester, they were observed in several following pregnancies. Albeit gestation by itself may be associated with primary hyperparathyroidism of pregnancy, such a situation probably did not take place in the described patient. We think so because this complication did not develop in her last pregnancy, which occurred after removing the adenoma.

Although the treatment of choice in primary hyperparathyroidism is the surgical intervention performed preferably during the second trimester of pregnancy, the patient declined this option of treatment because of the fear of potential complications to the fetus $[12,13]$. Parathyroidectomy in pregnancy is associated with a slightly increased risk of a spontaneous abortion [12, 13]. Respecting the choice of the patient, we decided on calcitonin administration. This agent is considered the safest conservative treatment 
option in patients with hypercalcemia in pregnancy [5]. The safety of calcitonin during gestation probably results from its negligible passage through the placenta $[14,15]$. The choice of this agent was also supported by suggested but not fully supported beneficial effects of calcitonin in the management of pancreatitis observed in non-pregnant subjects [16]. Although presently no parameters are known that may predict the outcome of primary hyperparathyroidism, in order to limit the risk of serious complications, most professionals recommend maintaining total plasma calcium at the level lower than $3.0 \mathrm{mmol} / \mathrm{L}$ [17]. Exceeding this level we considered an indication for calcitonin administration. Interestingly, although calcitonin treatment may be associated with the development of tachyphylaxis [18], only tendency to tachyphylaxis was observed in our patient in the third trimester, and it disappeared after introduction of oral phosphates. This fact may be explained by administration of this agent only if plasma calcium levels exceeded the established threshold. Moreover, the patient did not experience nausea, vomiting, diarrhea, flushing, injection site reactions and any other side effects associated with calcitonin treatment [18]. Although some animal studies suggested low birth weight in offsprings of dams treated during pregnancy with high doses of calcitonin [16] this was not the case in our patient, whose infant's birth mass was within normal limits. Interestingly, recently Horjus et al. [17] have shown the benefits of combined administration of calcitonin and cinacalcet in pregnancy and puerperium. Because this treatment option was inaccessible for us, we cannot support the utility of this calcimimetic in hyperparathyroid pregnant women.

The only symptom experienced by the neonate after delivery was transient hypocalcemia, which disappeared shortly after the beginning of intravenous calcium administration. The presence of transient mild hypocalcemia, probably resulted from increased calcium levels in fetal plasma, inhibiting parathyroid PTH synthesis and release during the pregnancy $[14,15,19]$. Interestingly, neonatal hypocalcemia was absent in the patient's subsequent pregnancy, taking place after parathyroidectomy, which supports the recommendations that surgery should be considered the treatment of choice in young hyperparathyroid pregnant women or desiring pregnancy $[12,13]$. The effectiveness of surgical intervention in the prevention of fetal and neonatal complications clearly indicates that hyperparathyroidism, if successfully treated, cannot be regarded as a contraindication for consecutive pregnancies.

Summing up, the described case of our patient indicates that pregnant women with recurrent spontaneous abortions should be diagnosed for the presence of primary hyperparathyroidism. It also shows that in patients declining surgery, calcitonin, administered if needed, together with phosphates should be considered as a treatment option for this disorder, because of its effectiveness and accepted safety.

Conflict of interest The authors declare that they have no conflict of interest.

Open Access This article is distributed under the terms of the Creative Commons Attribution Noncommercial License which permits any noncommercial use, distribution, and reproduction in any medium, provided the original author(s) and source are credited.

\section{References}

1. Fraser WD (2009) Hyperparathyroidism. Lancet 374:145-158

2. Rodgers SE, Lew JI, Solórzano CC (2008) Primary hyperparathyroidism. Curr Opin Oncol 20:52-58

3. Silverberg SJ, Bilezikian JP (2006) The diagnosis and management of asymptomatic primary hyperparathyroidism. Nat Clin Pract Endocrinol Metab 2:494-503

4. Kovacs CS, El-Hajj Fuleihan G (2006) Calcium and bone disorders during pregnancy and lactation. Endocrinol Metab Clin North Am 35:21-51

5. Schnatz PF, Curry SL (2002) Primary hyperparathyroidism in pregnancy: evidence-based management. Obstet Gynecol Surv $57: 365-376$

6. Mestman JH (1998) Parathyroid disorders of pregnancy. Semin Perinatol 6:485-496

7. Murray JA, Newman WA 3rd, Dacus JV (1997) Hyperparathyroidism in pregnancy: diagnostic dilemma? Obstet Gynecol Surv 52:202-205

8. Kort KC, Schiller HJ, Numann PJ (1999) Hyperparathyroidism and pregnancy. Am J Surg 177:66-68

9. Kohlmeier L, Marcus R (1995) Calcium disorders of pregnancy. Endocrinol Metab Clin North Am 24:15-39

10. Inabnet WB, Baldwin D, Daniel RO, Staren ED (1996) Hyperparathyroidism and pancreatitis during pregnancy. Surgery 119:710-713

11. Dahan M, Chang RJ (2001) Pancreatitis secondary to hyperparathyroidism during pregnancy. Obstet Gynecol 98(5 Pt 2):923-925

12. Schnatz PF, Thaxton S (2005) Parathyroidectomy in the third trimester of pregnancy. Obstet Gynecol Surv 60:672-682

13. Pothiwala P, Levine SN (2009) Parathyroid surgery in pregnancy: review of the literature and localization by aspiration for parathyroid hormone levels. J Perinatol 29:779-784

14. Mitchell DM, Jüppner H (2010) Regulation of calcium homeostasis and bone metabolism in the fetus and neonate. Curr Opin Endocrinol Diabetes Obes 17:25-30

15. Kovacs CS, Kronenberg HM (1997) Maternal-fetal calcium and bone metabolism during pregnancy, puerperium and lactation. Endocr Rev 18:832-872

16. Wolfe HJ (1982) Calcitonin: perspectives in current concepts. J Endocrinol Invest 5:423-432

17. Horjus C, Groot I, Telting D, van Setten P, van Sorge A, Kovacs CS et al (2009) Cinacalcet for hyperparathyroidism in pregnancy and puerperium. J Pediatr Endocrinol Metab 22:741-749

18. Nussbaum SR (1993) Pathophysiology and management of severe hypercalcemia. Endocrinol Metab Clin North Am 22:343-362

19. Steffensrud S (2000) Parathyroids: the forgotten glands. Neonatal Netw 19:9-16 